\title{
Modelling of extended de-weight fuzzy control for an upper-limb exoskeleton
}

\author{
Siti Khadijah Ali ${ }^{1}$, Masnida Hussin ${ }^{2}$, M. Sukri Hadi ${ }^{3}$, M. Osman Tokhi ${ }^{4}$ \\ ${ }^{1,2}$ Faculty of Computer Science and Information Technology, Universiti Putra Malaysia, \\ 43400 UPM Serdang, Selangor, Malaysia \\ ${ }^{3}$ Faculty of Mechanical Engineering, Universiti Teknologi MARA Malaysia, \\ 40450 Shah Alam, Selangor, Malaysia \\ ${ }^{4}$ Department of Electrical Engineering, London South Bank University, \\ 103, Borough Rd, London SE1 0AA, United Kingdom \\ ${ }^{1}$ Corresponding author \\ E-mail: ${ }^{1}$ ctkhadijah@upm.edu.my, ${ }^{2}$ masnida@upm.edu.my, ${ }^{3}$ msukrihadi@uitm.edu.my, \\ ${ }^{4}$ tokhim@lsbu.ac.uk
}

Received 18 May 2020; received in revised form 3 September 2020; accepted 11 September 2020 DOI https://doi.org/10.21595/jve.2020.21481

Check for updates

Copyright $(C 2020$ Siti Khadijah Ali, et al. This is an open access article distributed under the Creative Commons Attribution License, which permits unrestricted use, distribution, and reproduction in any medium, provided the original work is properly cited.

\begin{abstract}
Performing heavy physical tasks, overhead work and long working hours are some examples of activities that can lead to musculoskeletal problems in humans. To overcome this issue, automated robots such as the upper-limb exoskeleton is used to assist humans while performing tasks. However, several concerns in developing the exoskeleton have been raised such as the control strategies used. In this study, a control strategy known as the extended de-weight fuzz was proposed to ensure that the exoskeleton could be maneuvered to the desired position with the least number of errors and minimum torque requirement. The extended de-weight fuzzy is a combination of the fuzzy-based PD and fuzzy-based de-weight controller systems. The extended de-weight fuzzy was then compared with the fuzzy-based PD and PID controllers, and the performances of these controllers were compared in terms of their deviations and required torques to perform tasks. The findings show that the proposed control strategy performs better than the fuzzy-based PD and PID controller systems.
\end{abstract}

Keywords: upper-limb exoskeleton, fuzzy-based de-weight, extended fuzzy-based de-weight, fatigue upper arm.

\section{Introduction}

The exoskeleton is one example of an assistive technological innovation. Nowadays, the use of an exoskeleton is not limited to assisting or augmenting human performance [1-4]. In fact, the exoskeleton has been identified as one of the solutions in dealing with muscle fatigue in humans [5-7]. Muscle fatigue can occur at any age group and it is a condition whereby the strength required to perform the tasks is greater than the motion strength of an individual $[8,9]$. Several factors that contribute to this problem are heavy physical activities, repetitive work, overhead work, and long working hours $[10,11]$. Muscle fatigue can affect the quality of life for workers and there is a high risk of experiencing musculoskeletal disease (MSD) that could degrade their work performance [4, 12-17].

An exoskeleton is classified into three parts, namely the upper-, lower-extremity, and whole-body suits $[8,18]$. There are several challenges in developing the exoskeleton, in which one of them is the development of the control system [19]. The control system is used to ensure that the exoskeleton is able to move along with the human upper limb [20-22]. In addition, the control system should be able to interact and respond to movements generated by humans. Since the development of the exoskeleton in this study is to assist humans when they are experiencing fatigue, it is thus essential for the exoskeleton to be able to identify the strength of humans.

The contributions of this work are summarised as follows:

- The proposed extended de-weight fuzzy controller can enhance the limitation of the 
fuzzy-based PD controller to ensure that the movements of the upper-limb exoskeleton are accurate.

- The proposed extended de-weight fuzzy controller provides an insight into the reduction of errors and torques for the upper-limb exoskeleton.

- The proposed extended de-weight fuzzy controller is also capable of handling disturbance.

\section{Research method}

The research method comprises the modelling of the human upper-limb and exoskeleton upper-limb. In the first section, the kinematics and dynamics of the upper-limb are presented and followed by the exoskeleton upper-limb.

\subsection{Kinematics and dynamics of the human arm}

The development of the upper-limb exoskeleton is based on the kinematics and dynamics of the human arm during daily activities. Hence, it is important to know how movement occurs in the human body, specifically the human upper limbs. It is known that the musculoskeletal system comprising the connective tissues, muscles, and joints supports, stabilises, protects, and produces precise movements for the human body.

The human upper limb is designed as segments of linked bones that are linked by joints that have multiple degrees of freedom. The movements of the human upper limb are due to the existence of muscles. Muscles provide power or moment across the joints to enable movement. Some examples of human movements are shoulder flexion/extension, shoulder abduction/adduction, elbow flexion/extension, and wrist flexion/extension.
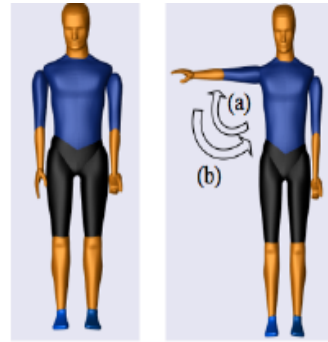

(1)

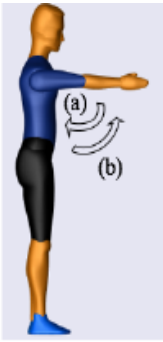

(2) (a)

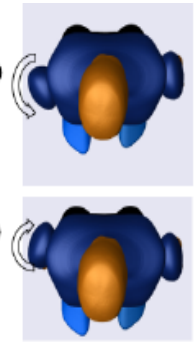

(3)

Fig. 1. Shoulder movements: Initial position (Extreme left), (1a) Shoulder abduction,

(1b) Shoulder adduction, (2a) Shoulder extension (2b), Shoulder flexion,

(3a) Shoulder internal rotation, and (3b) Shoulder external rotation

Table 1. Basic movements of the upper limb

\begin{tabular}{|c|c|c|c|c|c|c|c|}
\hline \multirow{2}{*}{ Joints } & \multicolumn{5}{|c|}{ Rotation } & \multicolumn{2}{c|}{ Translational } \\
\cline { 2 - 8 } & Flex/Ext & Abd/Add & Int/Ext & Pro/Sup & Rad/Ulnar & Up/Down & Fward/Bward \\
\hline Shoulder & $/$ & $/$ & $/$ & & & $/$ & $/$ \\
\hline Elbow & $/$ & & & & & & \\
\hline Wrist & $/$ & & & $/$ & $/$ & & \\
\hline
\end{tabular}

In this study, the development of the exoskeleton is solely based on the daily upper-extremity activities, in which the activities usually comprise combinations of basic movements. The movement combinations are presented in Table 1 and shown in Figs. 1-3. In this study, the shoulder is attached to the body by a ball and socket joint, and this joint is responsible for shoulder flexion/extension, shoulder adduction/abduction, and shoulder internal/external movements (Fig. 1). The upper and lower arm are connected by a single rotating joint known as a revolute joint at the elbow (Fig. 2). The lower arm and the palm are also connected by a revolute joint at the wrist (Fig. 3). The region of rotation for each joint is shown in Table 2. 

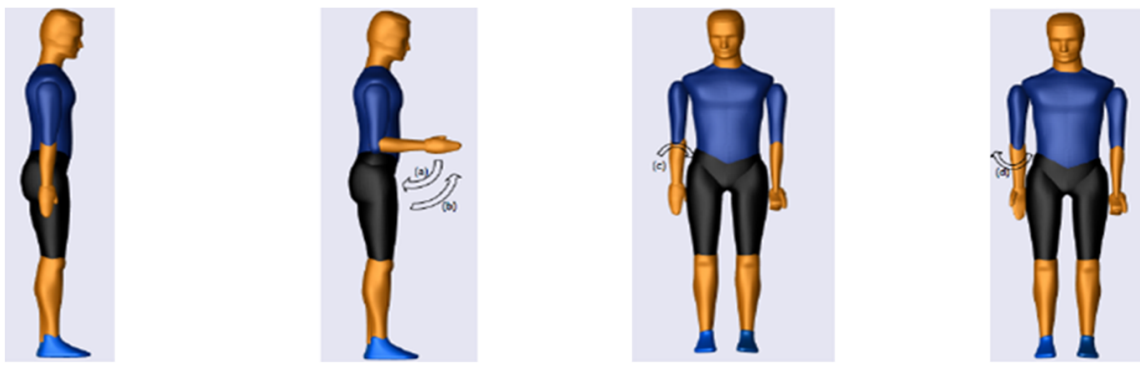

Fig. 2. Initial position (Extreme left) (a) Elbow flexion (b) Elbow extension (c) Forearm pronation

(d) Forearm supination
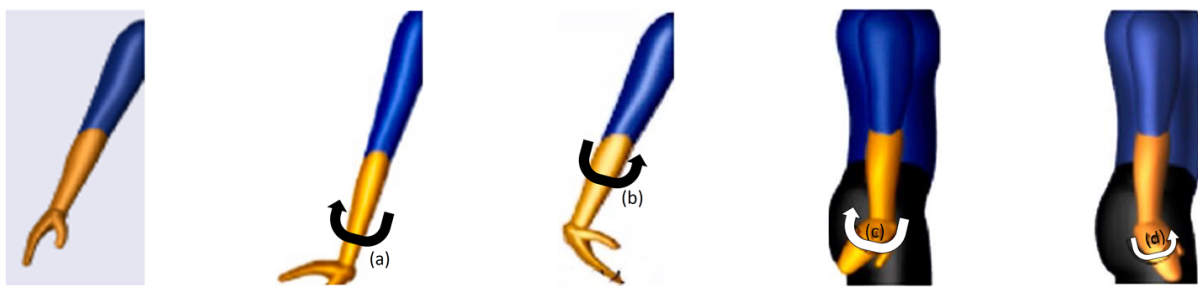

Fig. 3. Initial position (Extreme left) (a) Wrist extension (b) Wrist flexion (c) Ulnar deviation

(d) Radial deviation

Table 2. Range of motion and torques for human upper extremities [10, 23, 24]

\begin{tabular}{|c|c|c|}
\hline Joints & Range of motion & Torque, $\tau$ \\
\hline Shoulder flexion & $0-130^{\circ} / 180^{\circ}$ & $115^{\circ}$ \\
\hline Shoulder extension & $0-30^{\circ} / 80^{\circ}$ & $110^{\circ}$ \\
\hline Shoulder abduction & $0-180^{\circ}$ & $134^{\circ}$ \\
\hline Shoulder adduction & $0-50^{\circ}$ & $94^{\circ}$ \\
\hline Shoulder medial (Internal) & $0-60^{\circ} / 90^{\circ}$ & $39.2^{\circ}$ \\
\hline Shoulder lateral (External) & $0-90^{\circ}$ & $39.2^{\circ}$ \\
\hline Elbow flexion & $0-160^{\circ}$ & $72^{\circ}$ \\
\hline Elbow extension & $0-140^{\circ} / 146^{\circ}$ & $42^{\circ}$ \\
\hline Forearm pronation & $0-80^{\circ}$ & $9^{\circ}$ \\
\hline Forearm supination & $0-85^{\circ}$ & $7^{\circ}$ \\
\hline Wrist flexion & $0-90^{\circ}$ & - \\
\hline Wrist extension & $0-80^{\circ}$ & - \\
\hline Wrist abduction & $0-30^{\circ} / 40^{\circ}$ & - \\
\hline Wrist adduction & $0-150^{\circ}$ & - \\
\hline \multicolumn{2}{|c|}{} \\
\hline
\end{tabular}

\subsection{Mechanical design of the upper-limb exoskeleton}

The design of the exoskeleton used in this study was inspired by the TitanArm due to the simplicity of its design. Moreover, it is capable of powered use and data transmission on a mobile platform [9]. The material used to design the exoskeleton is aluminium as it is a low-density material with reasonable strength characteristics. As mentioned in Section 2.1, the shoulder joint in this study has three degrees of freedom, while the elbow and wrist joints have one degree of freedom, respectively (Fig. 4).

\subsubsection{Kinematics of the exoskeleton}

Fig. 5 shows the schematic diagram of the exoskeleton and the Denavit-Hartenberg Table for shoulder adduction/abduction movements. The $0_{1}, 0_{2}, 0_{3}$, and $0_{4}$ values represent the base for shoulder internal/external, shoulder extension/flexion, elbow extension/flexion, and the endpoint of the exoskeleton. The homogenous transformation matrix Eq. (1) is used to obtain the position 
and orientation of the end-effector $\left(0_{4}\right)$, with respect to the fixed reference frame $\left(0_{0}\right)$ :

$$
\begin{aligned}
T i_{i}^{i-1} & =\operatorname{Rot}\left(Z, \theta_{i}\right) \operatorname{Trains}\left(Z, d_{i}\right) \operatorname{Trains}\left(X, a_{i}\right) \operatorname{Rot}\left(X, \alpha_{i}\right) \\
& =\left[\begin{array}{cccc}
\cos \theta_{i} & \sin \theta_{i} \cos \alpha_{i} & \sin \theta_{i} \sin \alpha_{i} & a_{i} \cos \theta_{i} \\
\sin \theta_{i} & \cos \theta_{i} \cos \alpha_{i} & -\cos \theta_{i} \cos \alpha_{i} & a_{i} \sin \theta_{i} \\
0 & \sin \alpha_{i} & \cos \theta_{i} & d_{i} \\
0 & 0 & 0 & 1
\end{array}\right] .
\end{aligned}
$$

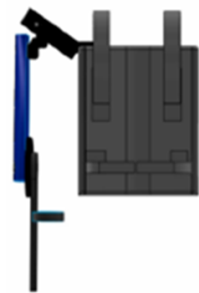

a) Front view

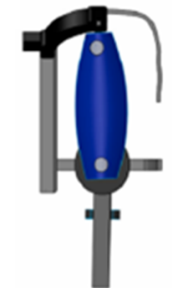

b) Right view

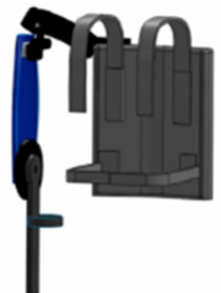

c) Perspective view

Fig. 4. View of the exoskeleton from three perspectives

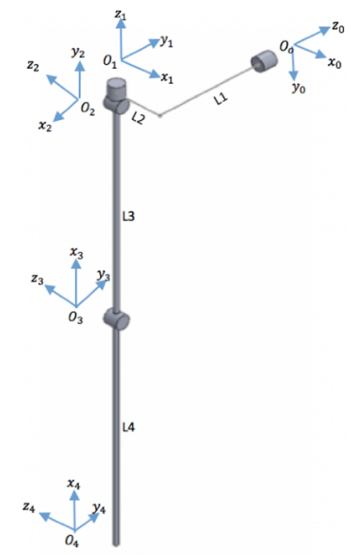

a)

\begin{tabular}{|c|c|c|c|c|}
\hline Frame & $\theta_{i}$ & $\alpha_{i}$ & $d_{i}$ & $a_{i}$ \\
\hline 1 & $\theta_{1}$ & $\frac{\pi}{2}$ & $L_{1}$ & $L_{2}$ \\
\hline 2 & $\theta_{2}-\frac{\pi}{2}$ & $\frac{\pi}{2}$ & 0 & 0 \\
\hline 3 & $\theta_{3}+\frac{\pi}{2}$ & 0 & 0 & $L_{3}$ \\
\hline 4 & $\theta_{4}$ & 0 & 0 & $L_{4}$ \\
\hline
\end{tabular}

b)

Fig. 5. a) Schematic diagram of exoskeleton, b) Denavit-Hartenberg table

\subsubsection{Dynamics of the exoskeleton}

The dynamics of the upper-limb exoskeleton was developed using the Euler-Lagrange approach as it is frequently used for the modelling of rigid robotic systems. Euler Lagrange is derived from Lagrangian $(\mathcal{L})$. Lagrangian is the difference between the kinetic $(\mathcal{T})$ and potential $(\mathcal{V})$ energy:

$\mathcal{L}\left(q_{i}, \dot{q}_{i}\right)=\mathcal{T}\left(q_{i}, \dot{q}_{i}\right)-\mathcal{V}\left(q_{i}\right)$

The total kinetic and potential energy for the whole system is presented as follows:

$$
\begin{gathered}
\mathcal{T}\left(q_{i}, \dot{q}_{i}\right)=\frac{1}{2}\left(m_{1}\left(\dot{x}_{1}^{2}+\dot{y}_{1}^{2}\right)+m_{2}\left(\dot{x}_{2}^{2}+\dot{y}_{2}^{2}\right)+m_{3}\left(\dot{x}_{3}^{2}+\dot{y}_{3}^{2}\right)+m_{4}\left(\dot{x}_{4}^{2}+\dot{y}_{4}^{2}\right)\right) \\
+\frac{1}{2}\left(I_{1} \dot{\theta}_{1}^{2}+I_{2}\left(\dot{\theta}_{1}+\dot{\theta}_{2}\right)^{2}+I_{3}\left(\dot{\theta}_{1}+\dot{\theta}_{2}+\dot{\theta}_{3}\right)^{2}+I_{4}\left(\dot{\theta}_{1}+\dot{\theta}_{2}+\dot{\theta}_{3}+\dot{\theta}_{4}\right)^{2}\right. \\
\mathcal{V}\left(q_{i}\right)=m_{1} g y_{1}+m_{2} g y_{2}+m_{3} g y_{3}+m_{4} g y_{4} .
\end{gathered}
$$


By applying the partial derivative to Eqs. $(3,4)$, the dynamic of the whole system is obtained and presented as follows:

$$
M(q) \ddot{q}+C(q, \dot{q}) \dot{q}+G(q)=\tau .
$$

The $M(q)$ is the exoskeleton inertia matrix, $C(q, \dot{q})$ is a matrix containing Coriolis and Centrifugal terms, $G(q)$ is a vector containing the gravity term, and $\tau$ is the vector of external torques acting on the actuated degree-of-freedom (DOF). The $q, \dot{q}$, and $\ddot{q}$ are positions, angular velocities, and angular accelerations of the revolute joints.

The dynamic model of the whole system was validated by comparing the generated torques between Eq. (5) with the SimMechanics model (Fig. 6). The input to the system is the angular positions of the shoulder and elbow joints for both the flexion and extension movements. The results for the validation process are presented in Section 4.

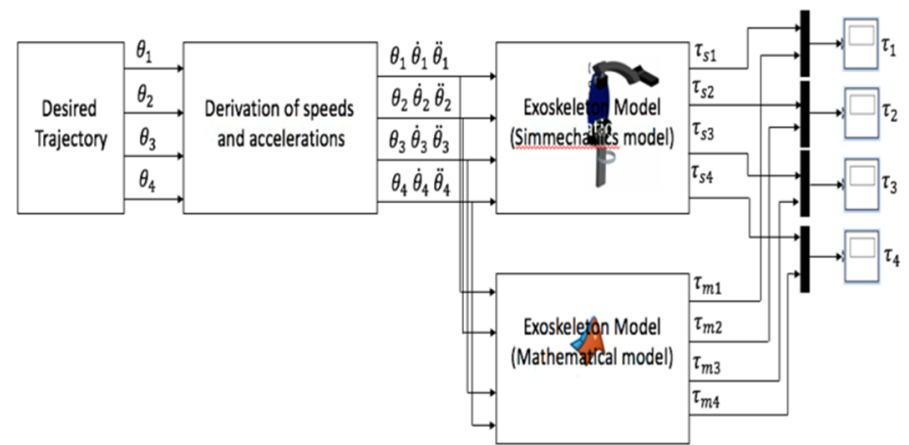

Fig. 6. Validation approach

\section{Control strategy}

The control design of this study is presented in Fig. 7. A detailed description of the control design is provided in $[1,2]$.

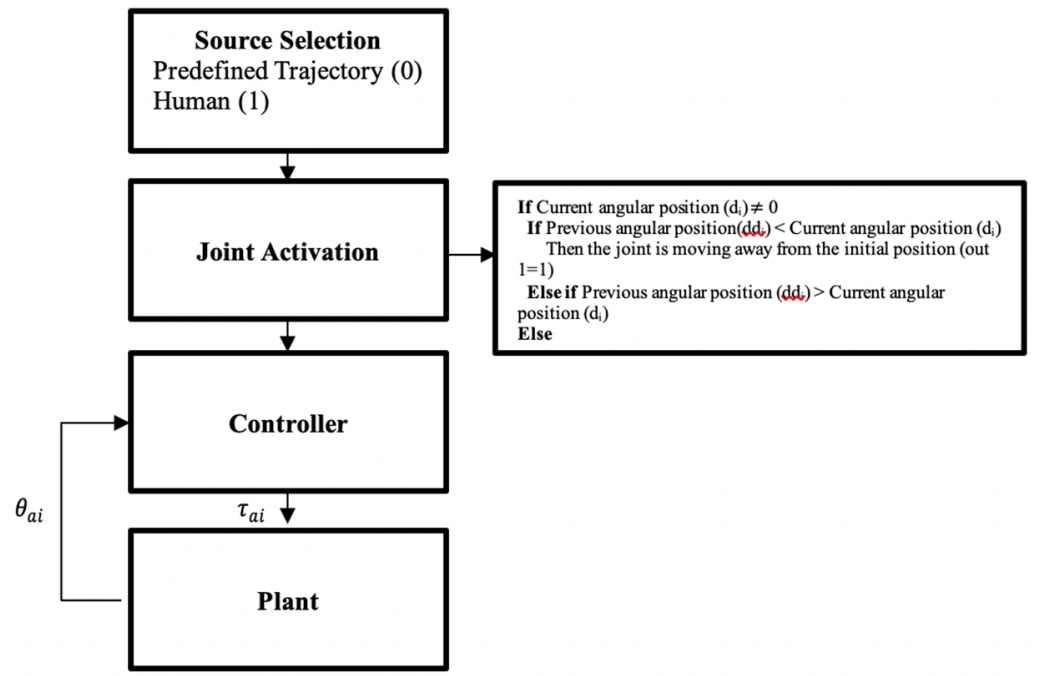

Fig. 7. Control design of the upper-limb exoskeleton

The proposed controller has been previously tested on humans with different strength [1]. In this study, the proposed controller was evaluated with the inclusion of disturbance. The 
performance of the proposed controller was compared with PID and fuzzy-based PD control. In the next section, the implementation of the PID, fuzzy-based PD, and the de-weight fuzzy controllers are described.

\subsection{Implementation of PID control}

The PID control was selected as a reference point for comparison as it was simple to implement and reliable. The input to the PID controller is the error of the trajectory. The error is obtained by comparing the actual trajectory from the desired trajectory. The error is then fed into the controller and the necessary torque is generated by the controller. The generated torque is then sent to the motor joint to move or to achieve the desired trajectory (Fig. 8).

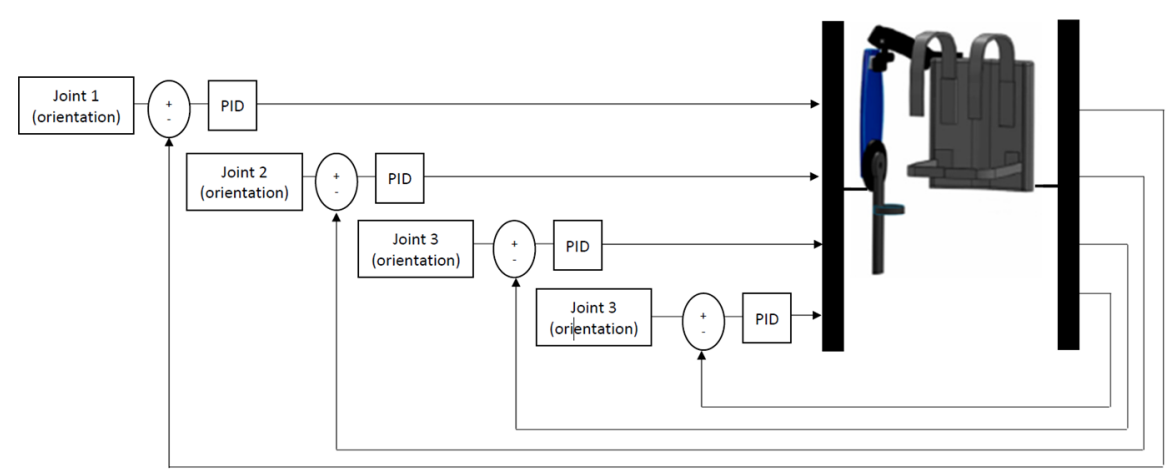

Fig. 8. Implementation of the exoskeleton with PID control

\subsection{Implementation of fuzzy-based PD control}

The implementation of the fuzzy-based PD control for the exoskeleton is presented in Fig. 9. The combination of fuzzy-based PD was selected due to the ability of PD to minimise the steady-state error and the rise time, thus requiring less power consumption.

The inputs to the fuzzy-based PD control are the trajectory error $(e)$ and the rate of change of error $(\dot{e})$. The error is obtained by measuring the difference between the actual trajectory and the desired trajectory, while the rate of change of error is calculated by the derivative of the error. The controller will generate the torque according to the inputs to ensure that the motor joint moves to the desired trajectory.

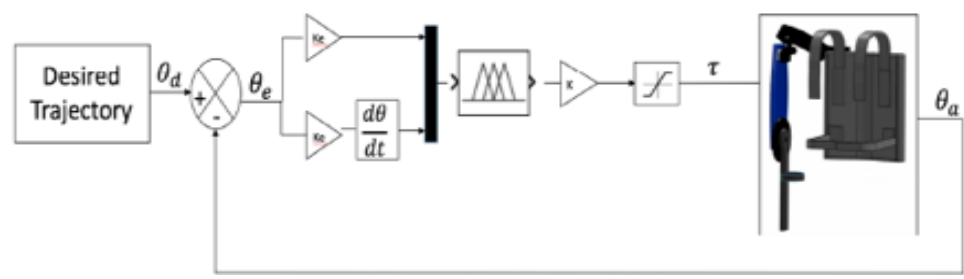

Fig. 9. Implementation of the exoskeleton with fuzzy-based PD control

\subsection{Implementation of an extended de-weight fuzzy control}

The extended de-weight fuzzy controller consists of fuzzy-based PD and fuzzy-based deweighting control (Fig. 10). The fuzzy-based PD assists the motor joint to move to the desired trajectory, whilst the fuzzy-based de-weighting is used to compensate the gravity torque to increase the smoothness of the movement. The inputs for the fuzzy-based PD is similar to those described in Section 3.2. The inputs for the fuzzy-based de-weight are position error $(e)$ and the current position of the motor joint $(a)$. The torques generated from the fuzzy-based PD and 
fuzzy-based de-weight are summed up and sent to the motor joint. For both fuzzy-based PD and extended de-weight fuzzy, a saturation block is included to ensure that the inserted torque is within the range of the human torque $[22,23]$.

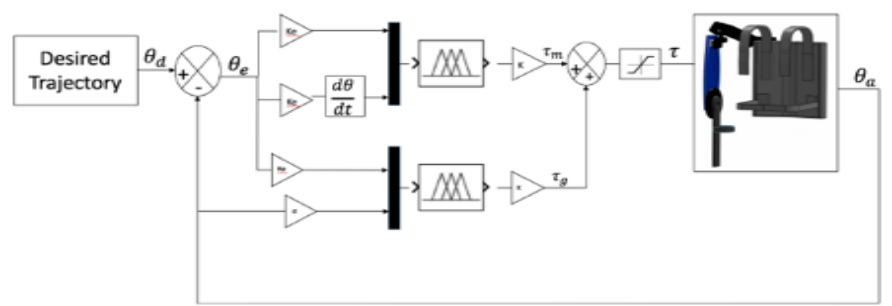

Fig. 10. Implementation of the exoskeleton with extended de-weight fuzzy control

\section{Results and discussion}

The experiments and simulations were performed using Simulink and SimMechanics. A computer with an Intel Core i7@2.6GHz processing system with 4G RAM was used to run the experiments and simulations.

\subsection{Validation of the dynamic model}

As previously described in Section 2.2.2, the inputs to the system were the angular positions of the shoulder and elbow joint. Flexion and extension movements were applied to both joints (Fig. 11).

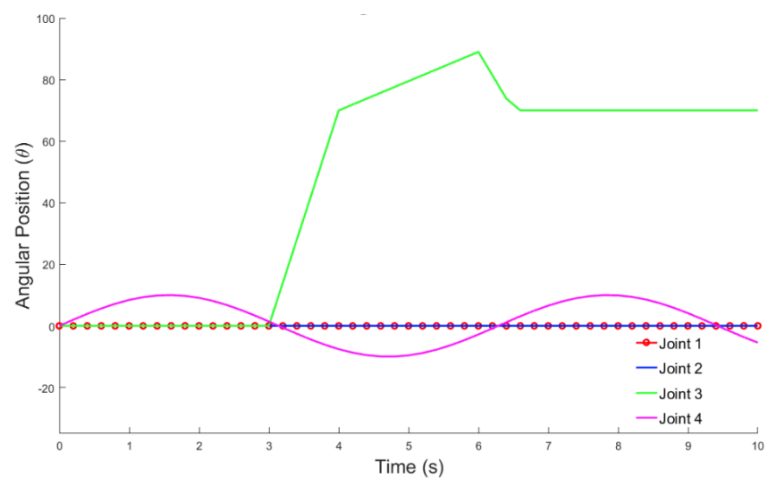

Fig. 11. Angular positions of flexion and extension for shoulder and elbow joints

Fig. 12(a-b) shows that although the movement patterns of the velocity and torque required for each joint were similar, they had different values. The torque generated from the mathematical representation (Simulink) was slightly higher than the value obtained from SimMechanics. This could be due to minor differences in the geometrical model built into SimMechanics and parameters described in the mathematical equation [10].

\subsection{Observation of control strategies}

As previously mentioned in Section 3, three types of controllers were used, and their performances were compared. During the experiments, a disturbance was included to validate the stability and robustness of the system. The external forces or disturbance at $1000 \mathrm{Nm}$ were applied at the position shown in Fig. 13 from $2.5 \mathrm{~s}$ until $10 \mathrm{~s}$. The external force applied at the forearm was expected to affect the movement of the elbow joint. 

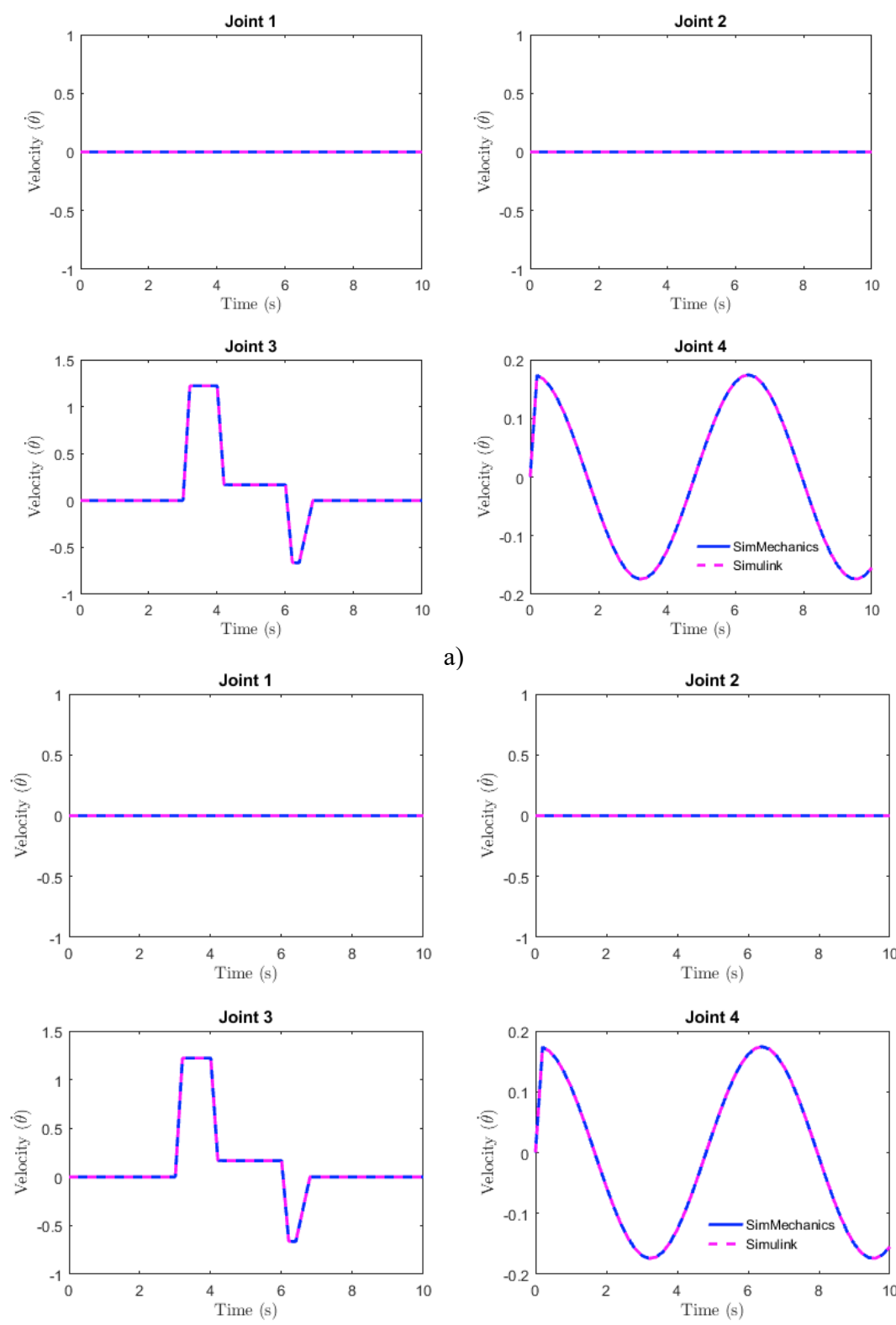

b)

Fig. 12. Flexion and extension movements: a) velocity and b) torque required

The control parameters used for PID, fuzzy-based PD, and extended de-weight fuzzy are shown in Table 3. These parameters were obtained by heuristic tuning. In general, all the controllers were able to track the desired movements even if a high disturbance was applied to the forearm of the exoskeleton (Figs. 14-16), thus indicating that all the controllers were stable and robust. As mentioned previously, joint 4 was the most affected as the disturbance was applied at the forearm. The observations were performed in two stages. Firstly, the PID and fuzzy-PD controllers in terms of root mean square (RMS) and maximum absolute error (MAE) (Table 4) were observed. As shown in Table 4, the RMS of Joint 4 for PID was slightly higher as compared to fuzzy-PD. In addition, the MAE of PID was almost $50 \%$ higher than the fuzzy-PD. This indicates that the maximum difference between the desired and actual trajectory for fuzzy-PD is 
better than PID, despite the high disturbance applied to the system.

Secondly, the fuzzy-PD was compared with the control proposed in this study, namely the extended de-weight fuzzy.

Table 3. Control parameters for PID, fuzzy-PD, and extended-de-weight fuzzy systems

\begin{tabular}{|c|c|c|c|c|}
\hline Control parameters & Joint 1 & Joint 2 & Joint 3 & Joint 4 \\
\hline$K_{p}$ & 10 & 1.0 & 10 & 100 \\
\hline$K_{I}$ & 5.0 & 1.0 & 5.0 & 70 \\
\hline$K_{D}$ & $1.0 e^{-1}$ & $1.0 e^{-1}$ & 0.1 & 4.0 \\
\hline$K_{e}$ & $2.0 e^{-2}$ & $1.0 e^{-4}$ & $1.0 e^{-2}$ & 1.0 \\
\hline$K_{\dot{e}}$ & $2.5 e^{-5}$ & $1.0 e^{-6}$ & $1.0 e^{-6}$ & 0 \\
\hline$K$ & 1000 & 1000 & 500 & 500 \\
\hline$K_{e}$ & $2.0 e^{-2}$ & $1.0 e^{-4}$ & $1.0 e^{-2}$ & 1.0 \\
\hline$K_{\dot{e}}$ & $2.5 e^{-5}$ & $1.0 e^{-6}$ & $1.0 e^{-6}$ & 0 \\
\hline$K_{1}$ & 1000 & 500 & 500 & 500 \\
\hline$K_{e}$ & 1.0 & 1.0 & 5.0 & 20.0 \\
\hline$K_{a}$ & $1.0 e^{-4}$ & $1.0 e^{-4}$ & $1.0 e^{-3}$ & $1.0 e^{-3}$ \\
\hline$K_{1}$ & 5 & 50 & 3 & 500 \\
\hline & & & &
\end{tabular}

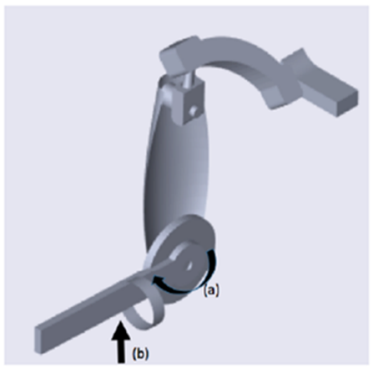

Table 4. RMS and MAE of Joint 4

\begin{tabular}{|c|c|c|}
\hline Controller/Joint & RMS & MAE \\
\hline PID & 0.3825 & 1.598 \\
\hline Fuzzy -PD & 0.3386 & 0.8735 \\
\hline Extended de-weight & 0.01802 & 0.03872 \\
\hline
\end{tabular}

Fig. 13. Exoskeleton: a) exoskeleton movement and b) external disturbance
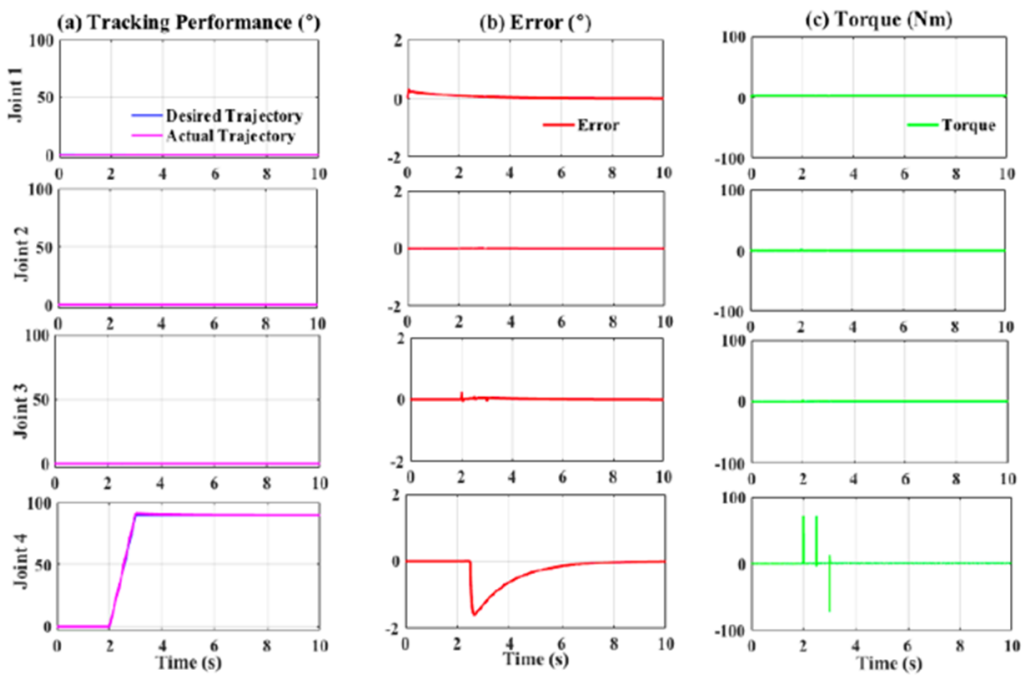

Fig. 14. Performance of PID controller with external force (1000 Nm)

Based on Table 4, the RMS and MAE values for the extended de-weight fuzzy were lesser as compared to fuzzy-PD. This indicates that the combination of fuzzy-based PD and de-weight fuzzy provides a more stable and robust system as compared to the previous study $[1,2]$. This 
observation could be due to the algorithm of the extended de-weight fuzzy itself, in which the value of the actual movements and the error is considered and subsequently added as inputs to the fuzzy. Therefore, when the disturbance was applied, the system could identify that there was a slight uncommon movement from the actual trajectory and hence, corrected the uncommon movement.
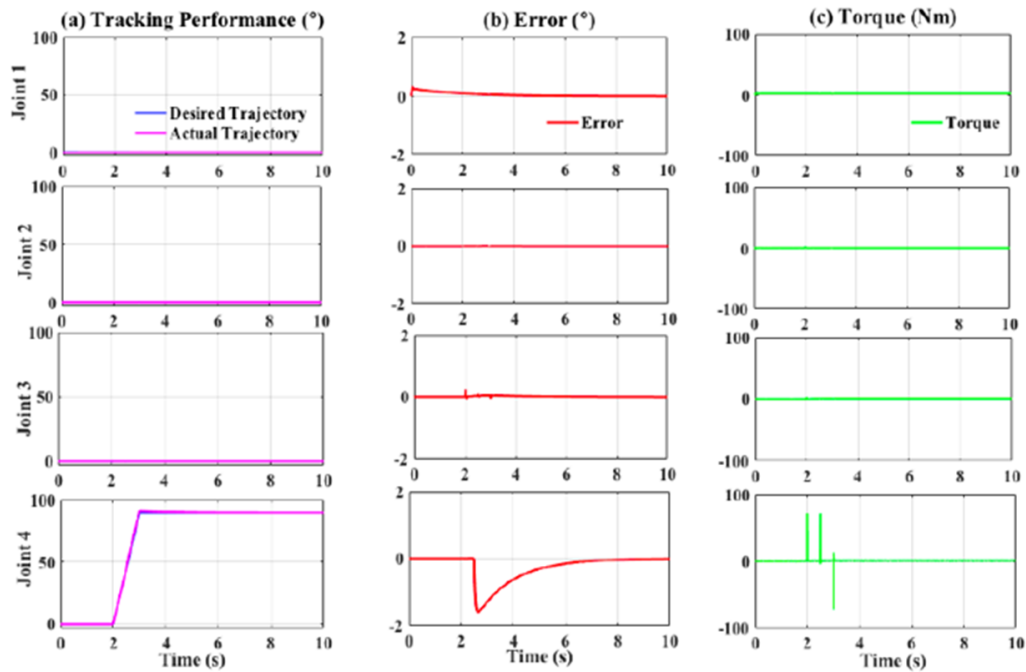

Fig. 15. Performance of fuzzy-PD controller with external force $(1000 \mathrm{Nm})$
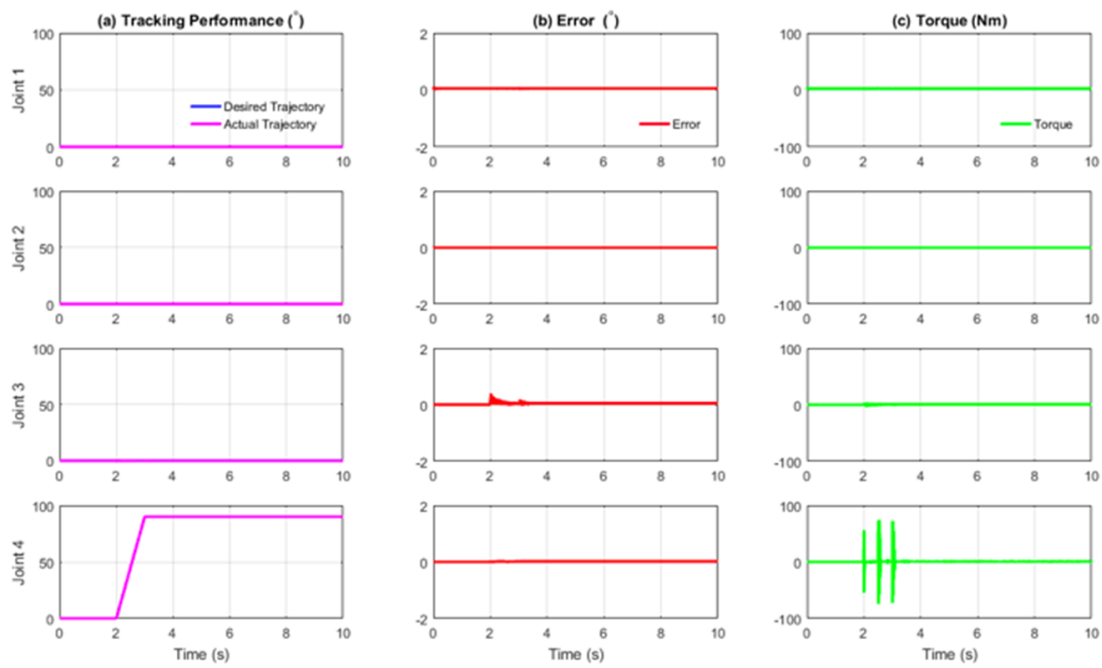

Fig. 16. Performance of extended de-weight fuzzy controller with external force $(1000 \mathrm{Nm})$

\section{Conclusions}

In this study, an extended de-weight control approach of an upper-limb exoskeleton with control saturation was developed for the joint angle position tracking objective. The de-weight fuzzy control was added to the fuzzy-based PD to compensate for the gravity torque to increase the smoothness of the movement. The robustness of the system was evaluated in this study. Simulation experiments including a comparison with PID and fuzzy-based PD controllers were performed, in which the proposed approach demonstrated satisfactory tracking performance and interaction torque reduction when high amounts of disturbance were applied. Based on these 
aspects, the performance of the proposed controller outperforms PID and fuzzy-based PD controller. In future experiments, the human fatigue model will be included to observe the robustness of the proposed control system.

\section{Acknowledgements}

This work was supported by Universiti Putra Malaysia and the Malaysian Ministry of Education through Geran Putra - Inisiatif Putra Muda (GP-IPM) (Vot: 9671000) and FRGS/1/2019/ICT02/UPM/02/3.

\section{References}

[1] Ali S. K., Tokhi M. O. Control design of a de-weighting upper-limb exoskeleton: extended-based fuzzy. Indonesian Journal of Electrical Engineering and Informatics (IJEEI), Vol. 7, Issue 1, 2019, p. 1-14.

[2] Ali S. K., Tokhi M. O. Control design of a de-weighting upper limb exoskeleton. International Conference on Applied Engineering (ICAE), 2018.

[3] Ali S. K., Tokhi M. O. Upper-limb exoskeleton for human muscle fatigue. Human-Centric Robotics: 20th International Conference on Climbing and Walking Robots and the Support Technologies for Mobile Machines, Porto, Portugal, 2017, p. 56-62.

[4] Ambrosini E., Ferrante S., Rossini M., Molteni F., Gföhler M., Reichenfelser W., Pedrocchi, A. Functional and usability assessment of a robotic exoskeleton arm to support activities of daily life. Robotica, Vol. 32, Issue 8, 2014, p. 1213-1224.

[5] Fan L., Yan L., Xiao J., Wang F. Dynamics analysis and simulation verification of a novel knee joint exoskeleton. Journal of Vibroengineering, Vol. 19, Issue 4, 2017, p. 3008-3018.

[6] Ochoa Luna C., Habibur Rahman M., Saad M., Archambault P. S., Bruce Ferrer S. Admittancebased upper limb robotic active and active-assistive movements. International Journal of Advanced Robotic Systems, Vol. 12, Issue 9, 2015, p. 117.

[7] Theurel J., Desbrosses K., Roux T., Savescu A. Physiological consequences of using an upper limb exoskeleton during manual handling tasks. Applied Ergonomics, Vol. 67, 2018, p. 211-217.

[8] Anam, Al Jumaily A.-A. Active exoskeleton control systems: state of the art. Procedia Engineering, Vol. 41, 2012, p. 988-994.

[9] Beattie E., Mcgill N., Parrotta N., Vladimirov N. Titan: a Powered, Upper Body Exoskeleton. Retrieved November 22, 2014.

[10] Biesiacki P., Mrozowski J., Awrejcewicz J. Study of dynamic forces in human upper limb inforward fall. Dynamical Systems - Applications, Publishing House of Lodz University of Technology, 2015, p. 65-76.

[11] Bosch T., Van Eck J., Knitel K., De Looze M. The effects of a passive exoskeleton on muscle activity, discomfort and endurance time in forward bending work. Applied Ergonomics, Vol. 54, 2016, p. 212-217.

[12] Carmichael M. G., Liu D., Waldron K. J. Investigation of reducing fatigue and musculoskeletal disorder with passive actuators. IEEE/RSJ International Conference on Intelligent Robots and Systems, 2010, p. 2481-2486.

[13] Coffin C. T. The use of a vertical arm support device to reduce upper extremity muscle firing in sonographers. Work, Vol. 42, Issue 3, 2012, p. 367-371.

[14] Lalitharatne T. D., Hayashi Y., Teramoto K., Kiguchi K. A study on effects of muscle fatigue on EMG-based con trol for human upper-limb power-assist. IEEE 6th International Conference on Information and Automation for Sustainability, 2012, p. 124-128.

[15] Ma L., Chablat D., Bennis F., Zhang W., Guillaume F. A new muscle fatigue and recovery model and its ergonomics application in human simulation. Virtual and Physical Prototyping, Vol. 5, Issue 3, 2010, p. 123-137.

[16] Martinez F., Retolaza I., Pujana Arrese A., Cenitagoya A., Basurko J., Landaluze J. Design of a five actuated DoF upper limb exoskeleton oriented to workplace help. 2nd IEEE RAS \& EMBS International Conference on Biomedical Robotics and Biomechatronics, 2008, p. 169-174.

[17] Xu W., Chu B., Rogers E. Iterative learning control for robotic-assisted upper limb stroke rehabilitation in the presence of muscle fatigue. Control Engineering Practice, Vol. 31, 2014, p. 63-72. 
[18] De Looze M. P., Bosch T., Krause F., Stadler K. S., O'sullivan L. W. Exoskeletons for industrial application and their potential effects on physical workload. Ergonomics, Vol. 59, Issue 5, 2016, p. 671-681.

[19] Huang J., Huo W., Xu W., Mohammed S., Amirat Y. Control of upper-limb power assist exoskeleton using a human-robot interface based on motion intention recognition. IEEE Transactions on Automation Science and Engineering, Vol. 12, Issue 4, 2015, p. 1257-1270.

[20] Brahim B., Maarouf S., Luna C. O., Abdelkrim B., Rahman M. H. Adaptive iterative observer based on integral backstepping control for upper extremity exoskelton robot. 8th International Conference on Modelling, Identification and Control (ICMIC), 2016, p. 886-891.

[21] Brahim B., Rahman M. H., Saad M., Luna C. O. Iterative estimator-based nonlinear backstepping control of a robotic exoskeleton. International Journal of Mechanical, Aerospace, Industrial, Mechatronic and Manufacturing Engineering, Vol. 10, Issue 8, 2016, p. 1313-1319.

[22] Brahmi B., Saad M., Rahman M. H., Ochoa Luna C. Cartesian trajectory tracking of a 7 DOF exoskeleton robot based on human inverse kinematics. IEEE Transactions on Systems, Man, and Cybernetics: Systems, Vol. 49, Issue 3, 2017, p. 600-611.

[23] Carignan C., Tang J., Roderick S., Naylor M. A configuration-space approach to controlling a rehabilitation arm exoskeleton. 10th International Conference on Rehabilitation Robotics, 2017, p. 179-187.

[24] Gupta A., O'malley M. K. Robotic Exoskeletons for Upper Extremity Rehabilitation. I-Tech Education and Publishing, Vienna, Austria, 2007, p. 371-396.

[25] Glowiński S., Krzyżyński T., Pecolt, Maciejewski I. Design of motion trajectory of an arm exoskeleton. Archive of Applied Mechanics, Vol. 85, Issue 1, 2015, p. 75-87.

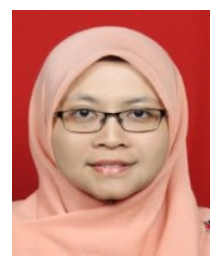

Siti Khadijah Ali is a senior Lecturer of the Department of Multimedia, Faculty of Computer Science and Information Technology, Universiti Putra Malaysia. She received her Ph.D. in automatic control and system engineering from University of Sheffield. Her research interests include computer graphics, physics-based animation/simulation and control system for exoskeleton.

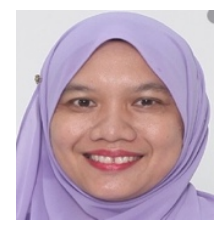

Masnida Hussin is aa Associate Professor of the Department of Communication Technology and Network, Faculty of Computer Science and Information Technology, Universiti Putra Malaysia. She received her Ph.D. in computer science from University of Sydney. Her research interests include resource management for large -scale parallel and distributed computing.

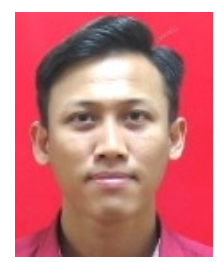

M. Sukri Hadi is a senior Lecturer of the Faculty of Engineering, Universiti Teknologi Mara Malaysia. He received his Ph.D. from Universiti Teknologi Malaysia. His research interests include artificial intelligence, evolutionary swarm algorithm, mechatronic, robotic, active vibration control.

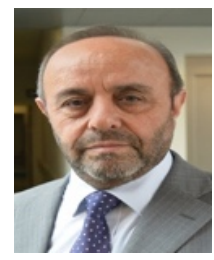

M. Osman Tokhi is a Professor of the Faculty of Engineering, London South Bank University, United Kingdom. He received his Ph.D. in Control Engineering from HeriotWatt University. His research interests include adaptive/intelligent computing and control, assistive robotics, active control of noise and vibration and high-performance real-time computing. 